\title{
Suppression of cell invasiveness by periostin via TAB1/TAK1
}

\author{
TAKAHIRO ISONO ${ }^{1}$, CHUL JANG KIM ${ }^{2}$, YUKIHIRO ANDO ${ }^{1,3}$, HIROAKI SAKURAI ${ }^{4}$, \\ YUSAKU OKADA ${ }^{5}$ and HIROKAZU INOUE ${ }^{6}$
}

\author{
${ }^{1}$ Central Research Laboratory, Shiga University of Medical Science, Setatsukinowa-cho, Otsu, Shiga 520-2192; \\ ${ }^{2}$ Department of Urology, Kohka Public Hospital, 3-39 Rokushin, Kohka, Shiga 528-0014; \\ ${ }^{3}$ Nagahama Institute of Bio-Science and Technology, Tamura 1266, Nagahama, Shiga 520-0829; \\ ${ }^{4}$ Division of Pathogenic Biochemistry, Institute of Natural Medicine, University of Toyama, Sugitani 2630, \\ Toyama 930-0194; ${ }^{5}$ Department of Urology, ${ }^{6}$ Division of Microbiology and Infectious Diseases, \\ Department of Pathology, Shiga University of Medical Science, Setatsukinowa-cho, Otsu, Shiga 520-2192, Japan
}

Received March 6, 2009; Accepted May 7, 2009

\section{DOI: 10.3892/ijo_00000355}

\begin{abstract}
We have previously shown that the expression of periostin is significantly downregulated in human bladder cancer tissues and that periostin suppresses cell invasiveness and metastasis of cancer cells. To clarify the molecular mechanism of this suppression by periostin, we searched for periostin-binding proteins and identified $\mathrm{TAB} 1$, which interacts with and activates TAK1, by mass analysis of proteins co-precipitated with periostin in $293 \mathrm{~T}$ cells expressing periostin. The association between periostin and TAB 1 was confirmed by a pulldown assay in 293T cells co-tranfected with expression plasmids of periostin, TAB 1 and TAK1. TAK1 was also co-precipitated with periostin in this assay. Co-transfection experiments in $293 \mathrm{~T}$ also showed that periostin could activate TAK1. Introduction of siRNA for TAB1 suppressed TAK1 activation by periostin. Analyses with deletion mutants of periostin revealed that the C-terminal region of periostin was necessary and sufficient for the association with TAB 1 and the TAK 1 activation. The suppression of invasiveness by periostin was attenuated by siRNA targeting TAK1 or TAB1 in 293T (human embryonic kidney) and T24 (human bladder carcinoma) cell lines. These findings indicate that periostin is involved in the suppression of cell invasiveness via the TAB1/TAK1 signaling pathway.
\end{abstract}

\section{Introduction}

Periostin has a typical signal peptide sequence at its $\mathrm{N}$-terminus and four repeated domains (RDs). Each of the latter contains

Correspondence to: Dr Hirokazu Inoue, Division of Microbiology and Infectious Diseases, Department of Pathology, Shiga University of Medical Science, Setatsukinowa-cho, Otsu, Shiga 520-2192, Japan

E-mail: hirokazu@belle.shiga-med.ac.jp

Key words: periosotin, TAB1, TAK1, invasion, bladder cancer two highly conserved sequences, which have been found in the fasciclin I family of proteins $(1,2)$. Periostin was initially shown to be highly expressed in early osteoblastic cells in vitro and in periosteum and periodontal ligament tissues in vivo, and it is known to play a role in bone and tooth formation and maintenance of structure in these tissues (1). Recently, it has been reported that periostin expression is correlated with heart failure (3-5), and that mechanical pressure stress induces the expression of periostin in fibroblasts in the heart (6) and in the periodontal ligaments (7). Periostin-null mice exhibit dwarfism, incisor enamel defects, and an early-onset periodontal disease-like phenotype, suggesting that periostin is required for maintenance of the integrity of the periodontal ligaments in response to mechanical stress (8).

It has also been reported that periostin expression is correlated with tumorigenesis (9). In human tumors, including colon cancer (10), breast cancer (11), lung cancer (12), head and neck cancer (13), oral cancer (14) and pancreatic cancer (15), periostin expression has been shown to be up-regulated; however, this is not true of bladder cancer (16). Recently, it was also shown in periostin-null mice that host-derived periostin negatively regulates tumor growth by promoting capsule formation (17). In previous studies, we have reported that the down-regulation of periostin mRNA expression is associated with higher grade in human bladder cancers, and that ectopic expression of periostin by a retroviral vector could suppress in vitro invasiveness of human bladder cancer cells and in vivo lung metastasis of mouse melanoma cells without affecting cell proliferation and tumor growth in nude mice (16). Our mutational analysis revealed that the C-terminal region of periostin, where alternative splicing events occur, is necessary and sufficient for the suppression of both in vitro cell invasiveness and in vivo lung metastasis (16). We have also demonstrated that the loss of wild-type periostin by down-regulation and/or alternative splicing in the C-terminal region is closely correlated with the development of bladder cancer (18). As for the molecular mechanism of regulation of tumor development by periostin, up-regulation of periostin has been shown to activate Akt to promote metastatic growth of colon cancer by preventing stress-induced apoptosis in cancer cells (10). Although periostin has been 
reported to act as a ligand for $\alpha_{v} \beta_{3}$ and $\alpha_{v} \beta_{5}$ integrins $(11,19)$, these results are based on indirect blocking experiments by anti-integrin antibodies. The target proteins directly associated with periostin to regulate tumor development have not been reported. In the present study, we attemped to identify the periostin-binding proteins to investigate the mechanism of suppression of cell invasiveness by periostin and found TAB1, an activator of TAK1 MAPKKK, as a binding protein of periostin. Furthermore, we demonstrated that periostin is able to activate TAK1 and to suppress in vitro cell invasiveness of bladder cancer cells via TAB1/TAK1.

\section{Materials and methods}

Cell lines. The human embryonic kidney cell line, 293T, and human bladder cancer cell line, T24, were cultured in Dulbecco's modified Eagle's medium (DMEM) supplemented with $10 \%$ fetal calf serum (FCS), penicillin $(100 \mathrm{U} / \mathrm{ml})$, and streptomycin $(100 \mu \mathrm{g} / \mathrm{ml})$ at $37^{\circ} \mathrm{C}$ in a humidified $5 \% \mathrm{CO}_{2}$ atmosphere.

Plasmids and transfection. The expression plasmid of FLAG-tagged human periostin was described previously (18). FLAG was conjugated to the C-terminus of periostin. Expression plasmids of HA-tagged rat wild-type (WT) periostin and three deletion mutants, the $\mathrm{N}$-terminal region deletion $(\Delta \mathrm{N}), \mathrm{C}$-terminal region deletion $(\Delta \mathrm{C})$, and $\mathrm{C}$-terminal region $(\mathrm{C}$-term), have been described previously $(16,20)$. The expression plasmid of human TAK 1 has been described previously (21). To construct the expression plasmid for TAB1 conjugated with Myc, we amplified $T A B 1$ gene fragments from T24 by PCR, using human TAB1-specific primers: TAB1S (5'-GATGGCGGCGCAGAGGAGGAG-3', the underlined nucleotide $2-4$ is the initial methionine codon) and TAB1A (5'-GGCTGCCCTACGGTGCTGTC-3', the underlined nucleotide $10-8$ is the stop codon). These amplified PCR fragments were cloned into Myc-tagged pcDNA3 expression vectors. DNA sequencing of the PCR products was performed by the dideoxy-chain termination method using an ABI PRISM 310 Genetic Analyzer (Applied Biosystems, Foster City, CA). All plasmids were prepared using Qiagen columns (Qiagen, Hiden, Germany). Transfection of plasmids was carried out in $60-\mathrm{mm}$ dishes, except for the experiments of identification of periostin-binding proteins, using Lipofectamine-PLUS reagent (Invitrogen, Carlsbad, CA) according to the manufacturer's protocol.

Preparation of recombinant retrovirus and virus infection. The pCXbsr retrovirus vectors carrying periostin cDNA (18) were transfected into $293 \mathrm{~T}$ cells with the helper plasmid pCL-ampho (22) using Lipofectamine-Plus reagent. The amphotropic retroviruses in the culture medium were collected $48 \mathrm{~h}$ after transfection, filtered, and stored at $-80^{\circ} \mathrm{C}$ until use. For the virus infection, $2 \times 10^{5}$ cells were plated onto $60-\mathrm{mm}$ dishes and were cultured overnight at $37^{\circ} \mathrm{C}$. After polybrene treatment $(2 \mu \mathrm{g} / \mathrm{ml})$ for $30 \mathrm{~min}$, the retroviruses were added to the cultures, which were then incubated for $1 \mathrm{~h}$ at $37^{\circ} \mathrm{C}$. After 7 days of incubation in selection medium (blasticidin $2 \mu \mathrm{g} / \mathrm{ml}$ ), the blasticidin-resistant colonies were pooled and used for assays.
In vitro invasion assay. The in vitro invasive potential of the cancer cells was determined using Matrigel ${ }^{\mathrm{TM}}$ Basement Membrane Matrix Invasion Chambers (chamber size, $6.4 \mathrm{~mm}$; membrane surface area, $0.3 \mathrm{~cm}^{2}$; pore size, $8 \mu \mathrm{m}$; BD Biosciences, Bedford, MA) following the manufacturer's instructions. Briefly, the culture supernatant used as a chemoattractant was prepared by culturing NIH3T3 cells in DMEM supplemented with $0.1 \%$ bovine albumin, $0.005 \% \mathrm{~L}(+)$ ascorbic acid, penicillin $(100 \mathrm{U} / \mathrm{ml})$ and streptomycin $(100 \mu \mathrm{g} / \mathrm{ml})$ at $37^{\circ} \mathrm{C}$ for $24 \mathrm{~h}$. Culture supernatant $(750 \mu \mathrm{l})$ was added to the plate well as a chemoattractant and $500 \mu \mathrm{l}$ of cell suspension at $5 \times 10^{4} 293 \mathrm{~T}$ cells $/ \mathrm{ml}$ or $2 \times 10^{4} \mathrm{~T} 24$ cells $/ \mathrm{ml}$ was added to each chamber. The chambers were incubated for 3 (293T) or 2 days (T24) in a humidified $5 \% \mathrm{CO}_{2}$ atmosphere. Noninvasive cells were removed from the upper surface of the membrane with a cotton swab. The invasive cells on the underside of the membrane were stained with Diff-Quik ${ }^{\mathrm{TM}}$ stain (Kokusaishiyaku, Kobe, Japan) and counted under a microscope. The Student's t-test was used for statistical analysis of the in vitro invasion assay. Only variables showing a value of $\mathrm{p}<0.05$ were considered statistically significant.

Antibodies. Anti-phospho-TAK1 (Thr187) antibody was generated by immunizing rabbits with the synthetic phosphopeptide corresponding to amino acids 180-194 of human TAK1 (23). Anti-c-Myc (no. 40, 9E10) antibody was purchased from Santa Cruz Biotechnology (Santa Cruz, CA). Anti-TAK1 (no. 7162) antibody was purchased from Cell Signaling (Danvers, MA). Anti-TAB1 antibody (AB051) was purchased from Kinasource Ltd. (Dundee, Scotland, UK). Anti- $\alpha$-tubulin (T9026, monoclonal) antibody and antiFLAG (M2, monoclonal) antibody were purchased from Sigma-Aldrich (St. Louis, MO). Anti-HA rat monoclonal antibody (3F10) was purchased from Roche Applied Science (Indianapolis, IN).

Immunoprecipitation. Cells were lysed in IP buffer containing $10 \mathrm{mM}$ Tris- $\mathrm{HCl}$ (pH 7.4), $150 \mathrm{mM} \mathrm{NaCl}, 1 \mathrm{mM}$ EDTA, $1 \mathrm{mM}$ EGTA, 1\% Triton-X, 0.5\% IGEPAL CA-630, 10\% glycerol, a protease inhibitor cocktail (Nakalai Tesque, Kyoto, Japan), and a phosphatase inhibitor cocktail (Nakalai Tesque), and were centrifuged for $30 \mathrm{~min}$ in $10,000 \mathrm{x} \mathrm{g}$ at $4^{\circ} \mathrm{C}$. For FLAGtagged proteins, the supernatant was incubated with antiFLAG affinity agarose (Sigma-Aldrich) at $4^{\circ} \mathrm{C}$ for $2 \mathrm{~h}$. For HA-tagged and Myc-tagged proteins, the supernatant was incubated with anti-HA or anti-Myc antibody at $4^{\circ} \mathrm{C}$ for $1 \mathrm{~h}$. The immunocomplexes were bound to protein- $\mathrm{G}$ sepharose for $1 \mathrm{~h}$ at $4^{\circ} \mathrm{C}$ and washed five times with IP buffer. The proteins bound to the resin were eluted by adding $2 \mathrm{X}$ Laemmli-SDS sample buffer, finally containing $62.5 \mathrm{mM}$ Tris- $\mathrm{HCl}$ (pH 6.8), $10 \%$ glycerol, 5\% 2-mercaptoethanol, $2 \%$ sodium dodecyl sulfate (SDS), and $0.01 \%$ bromophenol-blue, and boiling for $5 \mathrm{~min}$. After centrifugation at 10,000 x $\mathrm{g}$ for $2 \mathrm{~min}$, the supernatant was analyzed by immunoblotting.

Immunoblotting. Cells were lysed in Laemmli-SDS buffer, subjected to SDS-polyacrylamide gel electrophoresis, and electro-transferred to membrane filters (Immuno-Blot PVDF membranes, Bio-Rad Laboratories, Richmond, CA). The filters were incubated with a primary antibody in TBS-T 
(10 mM Tris- $\mathrm{HCl}, \mathrm{pH} 7.6,150 \mathrm{mM}$ sodium chloride, $0.1 \%$ Tween-20) containing $2 \%$ bovine serum albumin (BSA) overnight and incubated for $1 \mathrm{~h}$ in horseradish peroxidaseconjugated anti-mouse, anti-rabbit, anti-rat IgG (GE Healthcare, Buckinghamshire, UK), or anti-sheep IgG (SigmaAldrich) diluted 1:10,000 in TBS-T containing 2\% BSA. Immunoreactivity was detected by the ECL system (GE Healthcare) using LAS4000 (Fujifilm, Tokyo, Japan). Anti- $\alpha$ tubulin antibody was used as an internal control.

Small interfering RNAs (siRNA). siRNA targeting human TAK1 (L-003790-00-0005), TAB1 (L-004770-00-0005), and scramble control (D-001810-01-05) RNA duplexes were purchased from Dharmacon, Inc. (Lafayette, CO). Cells were transfected with RNA duplexes using Lipofectamine 2000 reagents (Invitrogen) following the manufacturer's protocol.

Identification of periostin-binding proteins by peptide-mass fingerprinting analyses. Peptide-mass fingerprinting analyses of periostin-binding proteins were performed as described previously (24) with minor modifications. 293T cells were transfected with the expression plasmid of FLAG-tagged periostin or vector plasmid (pCR3.1), and lysed in lysis buffer containing $20 \mathrm{mM}$ Tris- $\mathrm{HCl}$ ( $\mathrm{pH} 7.4), 150 \mathrm{mM} \mathrm{NaCl}, 5 \mathrm{mM}$ EDTA, 1\% Brij 98 (Sigma-Aldrich), a protease inhibitor cocktail (Nakalai Tesque) and a phosphatase inhibitor cocktail (Sigma-Aldrich). After the centrifugation of the cell lysates at $10,000 \mathrm{x}$, the supernatant was incubated with anti-FLAG affinity agarose (Sigma-Aldrich) at $4^{\circ} \mathrm{C}$ for $2 \mathrm{~h}$. Then the agarose was washed five times with lysis buffer and the proteins bound to the agarose were eluted by the FLAG peptide (150 $\mu \mathrm{M}$, Sigma-Aldrich). The samples were subjected to the SDS-PAGE gels and the proteins were visualized by silver staining. Each distinct band, which was detected only in cells expressing FLAG-tagged periostin, was digested in the gel by modified trypsin (Promega, Madison, WI). The extracted samples were analyzed by LC/MS/MS system with Paradigm MS4 (AMR, Tokyo, Japan), Finnigan LCQ Advantage (Thermo Fisher Scientific, Waltman, MA), and Bioworks analyzer soft (Thermo Fisher Scientific).

\section{Results}

Periostin associates with TAB1. To search for periostin-binding proteins, FLAG-tagged periostin protein was expressed in $293 \mathrm{~T}$ cells by transfection and immunoprecipitated with anti-FLAG affinity agarose. The proteins co-precipitated with periostin were analyzed by silver staining after SDS-PAGE in comparison with the lysate from the cells transfected with control plasmid (vector). As shown in Fig. 1A, a protein with molecular weight of about $60 \mathrm{kDa}$ was found as a candidate of periostin-binding proteins and was identified as TAB1 (TAK1 binding protein 1) by mass tag analyses using LC/MS/MS. TAB 1 is known to interact with and activate TAK1 (transforming growth factor-activated kinase), which is a ubiquitin-dependent kinase of MKK ( $\underline{\text { MAP kinase }}$ kinase) and IKK ( $\underline{\mathbf{I}} \underline{\mathrm{B}}$ kinase) and mediates various intracellular signaling pathways (25-27). To confirm the association between periostin and TAB1, we carried out a pulldown assay in $293 \mathrm{~T}$ cells transfected with the plasmid expressing periostin conjugated with HA (periostin-HA) and the expression plasmid of TAB1 conjugated with c-Myc (TAB1-Myc). To maintain the stability of TAB1 protein and assess the binding between periostin and TAK1, the plasmid expressing TAK1 (TAK1) was also co-transfected. As shown in Fig. 1B, TAB1-Myc was detected in the immunocomplex precipitated with anti-HA antibody and periostin-HA was also detected in the immunocomplex precipitated with the anti-Myc antibody in 293T cells expressing periostin-HA, TAB1-Myc and TAK1 proteins. This association was not detected when either periostin-HA or TAB1-Myc was absent. Co-transfected TAK1 was also detected in the immunocomplex precipitated with anti-HA antibody. Interestingly, the association of periostin-HA and TAK1 was detected without transfection of TAB1-Myc. These results indicate that periostin is able to form complex with TAB1 and TAK1. The same experiments were carried out with wild-type (WT) and deletion mutants of periostin $(\Delta \mathrm{N}$, $\Delta \mathrm{C}$, and C-term; see Fig. 1C). TAB1-Myc was co-precipitated with $\Delta \mathrm{N}$ and $\mathrm{C}$-term mutants of periostin, but was faintly coprecipitated with the $\Delta \mathrm{C}$ mutant (Fig. 1D). These results indicate that periostin is able to associate with $\mathrm{TAB} 1$ in its C-terminal region, which has been shown to be critical for suppression of cell invasiveness (16).

Activation of TAK1 by periostin. To investigate whether periostin is able to activate TAK $1,293 \mathrm{~T}$ cells were cotransfected with plasmids expressing TAK1, TAB1-Myc, and periostin-HA. Activation of TAK1 was monitored by immunoblotting with anti-phospho-TAK1 (Thr 187) antibody. As shown in Fig. 2A, co-transfection of periostin and TAK1 enhanced the phosphorylation of TAK1 compared to that of a single transfection by TAK1, although activation of TAK1 by periostin was weaker than that by TAB1. The expression of transfected TAK1 in 293T cells transfected with TAB1 was higher than that in $293 \mathrm{~T}$ cells transfected with periostin or vector plasmid, suggesting that TAB 1 contribute to enhance the stability of TAK 1 protein. This phenomenon was also observed in transfection experiment of Fig. 1B. In these experimental conditions, activation of TAK1 was not observed in 293T cells, which is not transfected with TAK1, probably because the expression level of endogenous TAK1 was very low in 293T cells as shown in Fig. 2A. The same experiments were carried out with deletion mutants of periostin $(\Delta \mathrm{N}, \Delta \mathrm{C}$, and $\mathrm{C}$-term). As shown in Fig. 2B, the C-term mutant enhanced the phosphorylation of TAK1 as well as WT periostin. Although the $\Delta \mathrm{N}$ mutant moderately promoted phosphorylation of TAK 1 , the $\Delta \mathrm{C}$ mutant hardly enhanced it. These results indicate that the $\mathrm{C}$-terminal region of periostin is necessary and sufficient for the activation of TAK1. This result agrees with our previous finding about the suppression of cell invasiveness by these mutants (16) and the results of binding assay between TAB1 and periostin mutants shown in Fig. 1D. To investigate whether TAB1 contributes to the activation of TAK1 by periostin, we performed a knockdown experiment using siRNA targeting human TAB1 in 293T cells co-transfected with TAK1 and periostin. Immunoblot analysis showed that expression of endogeneous TAB 1 was markedly reduced by introduction of siRNA targeting TAB1 but not by control siRNA (Fig. 2C). Co-transfection of siRNA targeting TAB1 attenuated phosphorylation of 


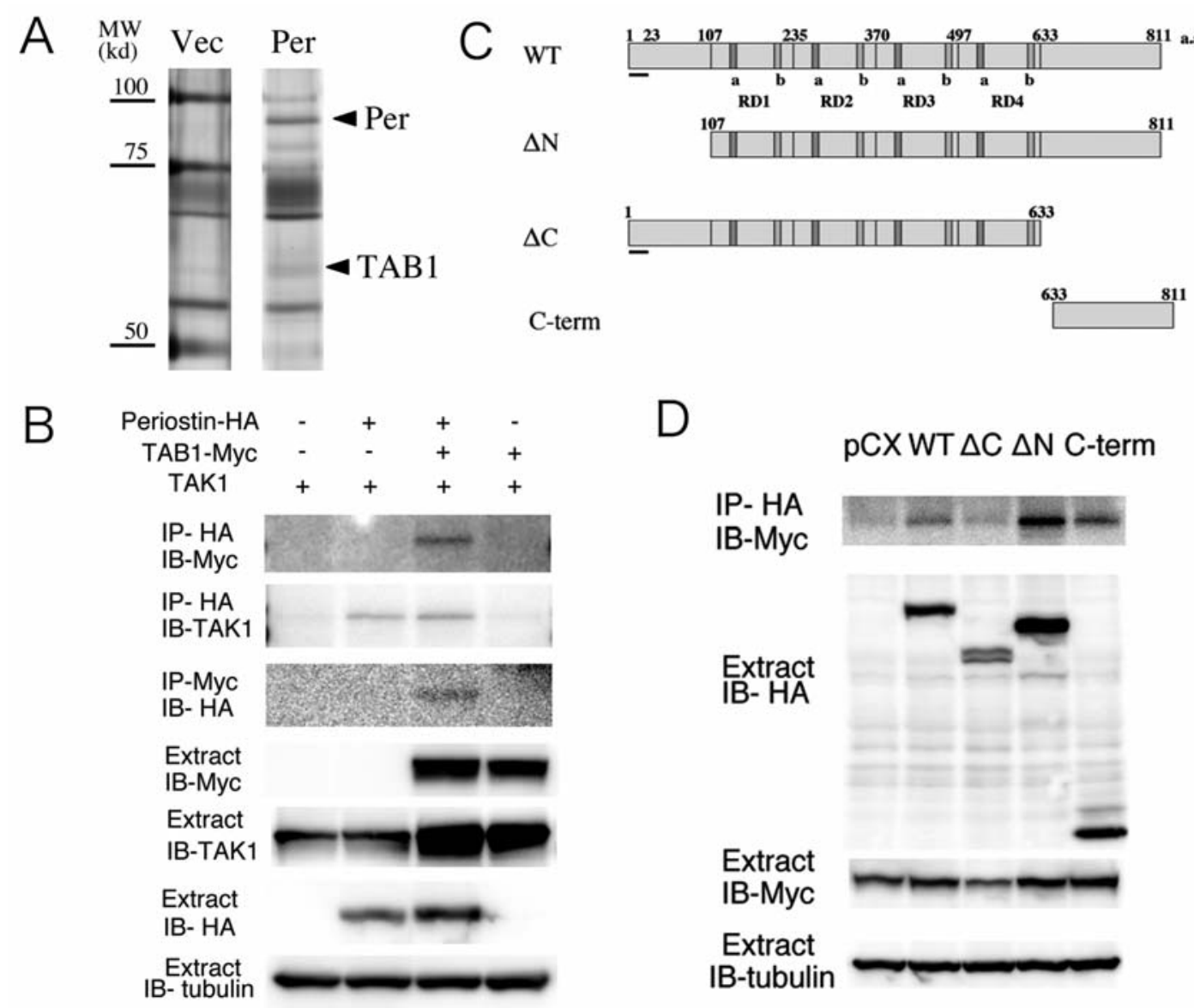

Figure 1. Association between periostin and TAB1. (A) Silver-stained image of SDS-PAGE gels of periostin-binding proteins. 293T cells transfected with pCR3.1 vector (Vec, left, $4 \mu \mathrm{g} / 100$-mm dish) or the expression plasmid of FLAG-tagged periostin (Per, right, $4 \mu \mathrm{g} / 100$-mm dish) was lysed and the proteins bound to anti-FLAG affinity agarose were eluted by FLAG peptide. Eluted proteins were analyzed by SDS-polyacrylamide electrophoresis followed by silver staining. Arrowheads (Per and TAB1) show periostin and TAB1 protein bands, respectively. (B) Association of periostin with TAB1 and TAK1 in $293 \mathrm{~T}$ cells. The expression plasmid of HA-tagged periostin $(2 \mu \mathrm{g})$ was co-transfected with the expression plasmid of Myc-tagged TAB1 $(0.2 \mu \mathrm{g})$ and TAK1 $(0.2 \mu \mathrm{g})$ in $293 \mathrm{~T}$ cells. As controls, pCR3.1 vector $(2 \mu \mathrm{g})$, the expression plasmid of HA-tagged periostin, and Myc-tagged TAB1 were each transfected with TAK1 into 293T cells. The cell lysates were immunoprecipitated with anti-HA or anti-Myc antibody and immunoblotted for anti-HA, anti-Myc, or anti-TAK1 antibody, respectively. To confirm the expression of each protein in transfected cells, whole lysed extracts were also immunoblotted for anti-Myc, anti-HA, and antiTAK1 antibodies. Tubulin was used as a loading control. IP and IB show immunoprecipitation and immunoblotting, respectively. (C) Structure of wild-type (WT) and deletion mutants ( $\Delta \mathrm{C}, \Delta \mathrm{N}$, and $\mathrm{C}$-term) of periostin. The bar at the $\mathrm{N}$-terminus represents the position of the typical signal peptide sequence. Each of the repeated domains (RDs) contains two highly conserved sequences that are indicated by boxes a and b. (D) Association of periostin deletion mutants with TAB1 in 293T cells. The expression plasmids of HA-tagged $\Delta \mathrm{C}(2 \mu \mathrm{g}), \Delta \mathrm{N}(3 \mu \mathrm{g})$, and C-term $(2 \mu \mathrm{g})$ were co-transfected with Myc-tagged TAB1 (0.2 $\mu \mathrm{g})$ and TAK1 $(0.2 \mu \mathrm{g})$ in $293 \mathrm{~T}$ cells. As negative and positive controls, an expression plasmid of the pCX vector $(2 \mu \mathrm{g})$ or HA-tagged wild-type (WT) periostin $(1 \mu \mathrm{g})$, respectively, was co-transfected with Myc-tagged TAB1 $(0.2 \mu \mathrm{g})$ and TAK1 $(0.2 \mu \mathrm{g})$ in 293T cells. Cell lysates were immunoprecipitated with antiHA antibody and immunoblotted for anti-Myc antibody. To confirm the expression of each protein, whole lysed extracts were also immunobloted for anti-HA and anti-Myc antibodies. Tubulin was used as a loading control. IP and IB show immunoprecipitation and immunoblotting, respectively.

TAK1 by periostin without affecting the expression of periostin. The level of TAK1 protein was also reduced by introduction of siRNA targeting TAB1, suggesting that $\mathrm{TAB} 1$ is required to maintain the stability of TAK1 protein. This result is consistent with co-transfection experiment in which the expression of TAB1 enhanced the expression level of transfected TAK1 protein (Fig. 2A). These results indicate that $\mathrm{TAB} 1$ is necessary for the activation of TAK1 by periostin in $293 \mathrm{~T}$ cells.

Periostin suppresses in vitro cell invasiveness via TAB1/TAK1 in 293 T cells. We have previously shown that periostin could suppress in vitro cell invasiveness of human bladder cancer cells $(16,18)$. To assess whether TAK1 contributes to the suppression of cell invasiveness by periostin, we investigated the effect of siRNA targeting human TAK1 in 293T cells. Immunoblot analysis showed that introduction of siRNA targeting TAK1 reduced the expression of endogenous TAK1 in 293T cells without affecting exogenous periostin expression (Fig. 3A). To quantitatively evaluate the invasive potential of 293T cells, we performed an in vitro invasion assay using Matrigel. As shown in Fig. $3 \mathrm{C}$ and D, the cell invasiveness of 293T cells transfected with periostin (Per) was markedly lower than that of control cells with the vector (pCX). Introduction of siRNA targeting TAK1 markedly inhibited the suppression of the cell invasiveness of 293T cells by periostin, but control siRNA did not. These results indicate that TAK1 is necessary for the suppression of cell invasiveness by periostin in 293T cells. To investigate whether the ectopic expression of TAK1 is able to suppress the cell 


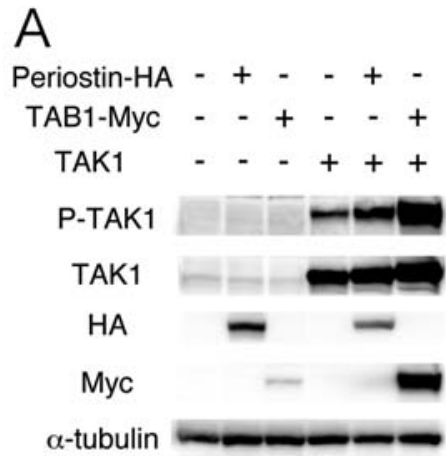

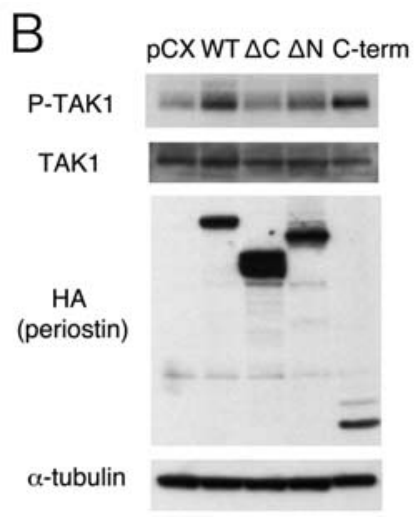

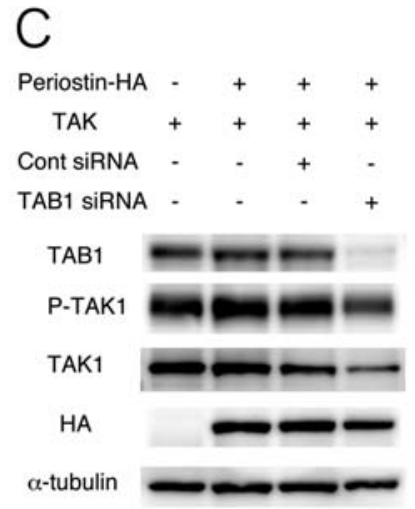

Figure 2. Activation of TAK1 by periostin. (A) Activation of TAK1 by periostin. The expression plasmid of TAK1 (0.2 $\mu \mathrm{g})$ was transfected with the expression plasmid of HA-tagged periostin ( $2 \mu \mathrm{g})$ or Myc-tagged TAB1 $(0.2 \mu \mathrm{g})$ in 293T cells. Activation of TAK1 was assayed by immunoblotting using anti-phosphoTAK1 (Thr187) antibody. To confirm the expression of each protein in transfected cells, whole lysed extracts were also immunoblotted for anti-TAK1, anti-Myc and anti-HA antibodies. Tubulin was used as a loading control. (B) Activation of TAK1 by deletion mutants of periostin. The expression plasmids of HA-tagged $\Delta \mathrm{C}$ $(2 \mu \mathrm{g}), \Delta \mathrm{N}(3 \mu \mathrm{g})$, and C-term $(2 \mu \mathrm{g})$ were co-transfected with TAK1 $(0.2 \mu \mathrm{g})$ in 293T cells. As controls, an expression plasmid of either the pCX vector $(2 \mu \mathrm{g})$ or HA-tagged wild-type (WT) periostin $(1 \mu \mathrm{g})$ was co-transfected with TAK1 $(0.2 \mu \mathrm{g})$ in 293T cells. Activation of TAK1 was assayed by immunoblotting using anti-phospho-TAK1 (Thr 187) antibody. To confirm the expression of each protein in transfected cells, whole lysed extract were also immunoblotted for anti-TAK1 and anti-HA antibodies. Tubulin was used as a loading control. (C) Effect of siRNA targeting of TAB1 on TAK1 activation by periostin in 293T cells. Activation of TAK1 was assayed by immunoblotting using anti-phospho-TAK1 (Thr 187) antibody. To confirm the expression of each protein in transfected cells, whole lysed extracts were also immunoblotted for anti-TAK1, anti-TAB1, and anti-HA antibodies. Tubulin was used as a loading control.
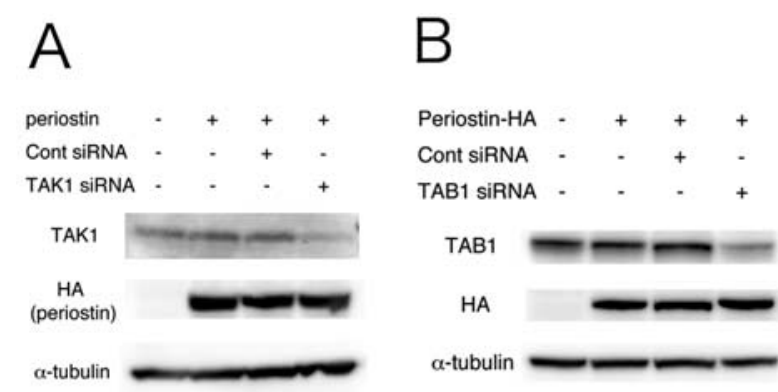

\section{C}

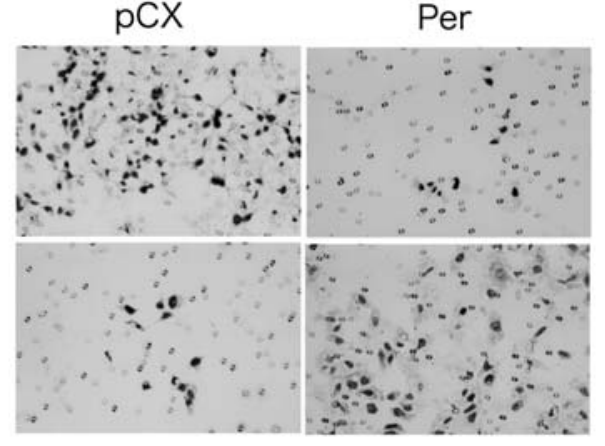

Per + cont siRNA Per + TAK siRNA

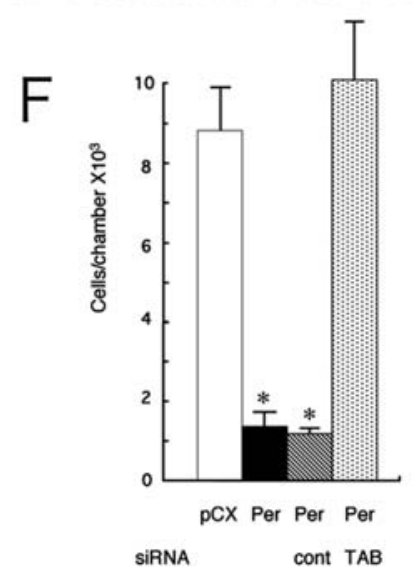

Figure 3. Effect of siRNA targeting human TAK1 or TAB1 on the suppression of cell invasiveness by periostin in 293T cells. (A) Effect of siRNA targeting TAK1 on the expression of endogenous TAK1 in 293T cells. The expression plasmid of HA-tagged periostin $(4 \mu \mathrm{g})$ was transfected singly, with control siRNA (120 pmole), and with siRNA targeting TAK1 (120 pmole) into 293T cells. As a control, pCR3.1 vector (4 $\mu \mathrm{g})$ was transfected in 293T cells. To confirm the expression of each protein in transfected cells, whole lysed extracts were immunoblotted for anti-TAK1 and anti-HA antibodies. Tubulin was used as a loading control. (B) Effect of siRNA targeting TAB1 on the expression of endogenous TAB1 in 293T cells. The expression plasmid of HA-tagged periostin (4 $\mu \mathrm{g})$ was transfected singly, with control siRNA (120 pmole), and with siRNA (120 pmole) targeting human TAB1 into $293 \mathrm{~T}$ cells. As a control, pCR3.1 vector (4 $\mu \mathrm{g})$ was transfected into 293T cells. To confirm the expression of each protein in transfected cells, whole lysed extracts were also immunoblotted for anti-TAB1 and anti-HA antibodies. Tubulin was used as a loading control. (C) Pictures of the cells invading through Matrigel. The procedures are described in Materials and methods. Photographs were taken at magnification x100. (D) The numbers of cells invading through the Matrigel in 293T cells. Each sample was assayed in triplicate. Bars $\pm \mathrm{SE}$ of triplicate chambers for each experiment. " $\mathrm{p}<0.05$; as compared to pCR3.1 vector and siRNA targeting TAK1. (E) Effect of ectopic overexpression of TAK1 on cell invasiveness of $293 \mathrm{~T}$ cells. The expression plasmid of TAK1 $(1 \mu \mathrm{g})$ was transfected into $293 \mathrm{~T}$ cells. As control, pCR3.1 vector $(2 \mu \mathrm{g})$ was transfected into 293T cells. The numbers of cells invading through the Matrigel in 293T cells are shown. Each sample was assayed in triplicate. Bars \pm SE of triplicate chambers for each experiment. "p $<0.05$; as compared to pCR3.1 vector. (F) The numbers of cells invading through the Matrigel in 293T cells. Each sample was assayed in triplicate. Bars $\pm \mathrm{SE}$ of triplicate chambers for each experiment. " $\mathrm{p}<0.05$; as compared to pCR3.1 vector and siRNA targeting TAB1. 


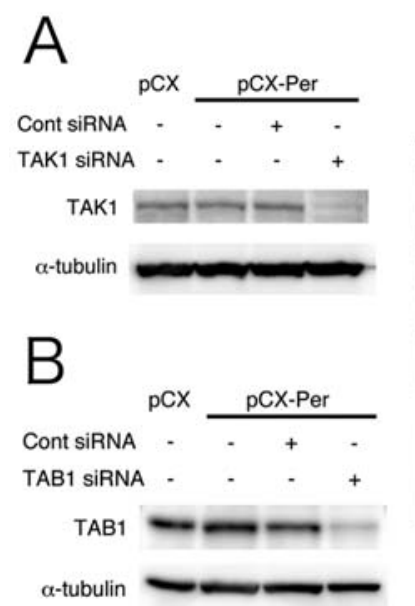

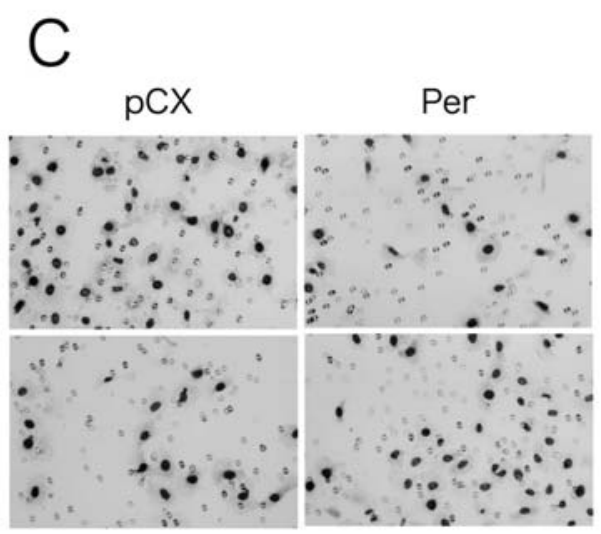

Per + cont siRNA Per + TAK siRNA
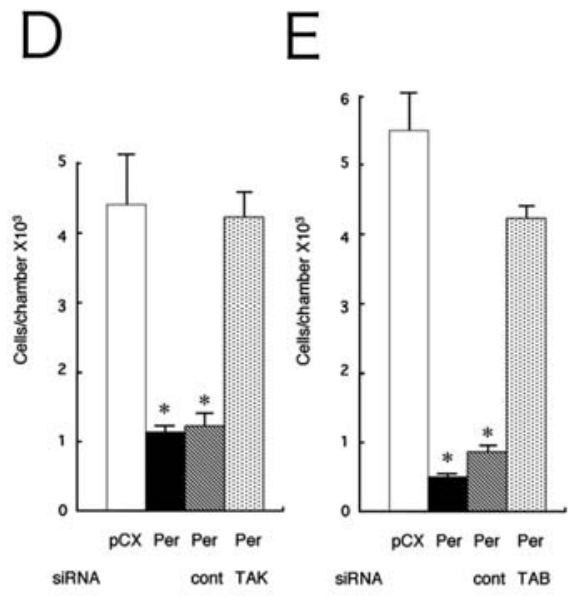

Figure 4. Effect of siRNA targeting human TAK1 or TAB1 on the suppression of cell invasiveness by periostin in T24 human bladder cancer cells. (A) Effect of siRNA targeting TAK1 on the expression of endogenous TAK1 in T24 cells. Control siRNA (240 pmole) or siRNA targeting TAK1 (240 pmole) was transfected into T24 cells infected with the recombinant retrovirus expressing periostin (pCX-Periostin). As controls, T24 cells infected with the recombinant retrovirus expressing periostin (pCX-Periostin) and with the control pCXbsr vector virus (pCX) were used. To examine the expression of TAK1 in transfected cells, whole lysed extract was immunoblotted for the anti-TAK1 antibody. Tubulin was used as a loading control. (B) Effect of siRNA targeting of TAB1 on the expression of endogenous TAB 1 in T24 cells. Control siRNA ( 240 pmole) or siRNA targeting TAB1 ( 240 pmole) was transfected into T24 cells infected with the recombinant retrovirus expressing periostin (pCX-Periostin). As controls, T24 cells infected with the recombinant retrovirus expressing periostin (pCX-Periostin) and with the control pCXbsr vector virus (pCX) were used. To examine the expression of TAB1 in transfected cells, whole lysed extract was immunoblotted for the anti-TAB1 antibody. Tubulin was used as a loading control. (C) Pictures of the cells invading through Matrigel. The procedures are described in Materials and methods. Photographs were taken at magnification x100. (D and E) The numbers of cells invading through the Matrigel in T24 cells. Each sample was assayed in triplicate. Bars \pm SE of triplicate chambers for each experiment. (D) *p<0.05; as compared to siRNA targeting TAK1. (E) * $\mathrm{p}<0.05$; as compared to the pCXbsr vector and siRNA targeting TAB1.

invasiveness of $293 \mathrm{~T}$ cells, we also carried out a Matrigel assay with the expression plasmid of TAK1. As shown in Fig. 3E, the cell invasiveness of 293T cells transfected with TAK1 was markedly lower than that of control cells with $\mathrm{pCX}$, indicating that the activation of TAK1 is critical for the suppression of cell invasiveness in 293T. In Fig. 2C, we showed that TAB1 is required for TAK1 activation by periostin. To examine whether TAB 1 contributes to the suppression of cell invasiveness by periostin, we performed the same knockdown experiment using siRNA targeting human TAB1 in 293T. Introduction of siRNA targeting TAB 1 reduced the expression of endogenous TAB1 in 293T cells without affecting periostin expression (Fig. 3B). As shown in Fig. 3F, introduction of siRNA targeting TAB1 also markedly inhibited the suppression of cell invasiveness by periostin as well as that by siRNA targeting TAK1. These results, together with the results of the knockdown experiment by TAK 1 siRNA, indicate that TAB1/TAK1 signaling is necessary for the suppression of cell invasiveness by periostin in $293 \mathrm{~T}$ cells.

Periostin suppresses cell invasiveness via TAB1 and TAK1 in human bladder cancer cells. To investigate whether TAB1 and TAK1 also contribute to the suppression of cell invasiveness by periostin in human bladder cancer cells, we performed knockdown experiments in a human bladder cancer cell line, T24, which lacks the expression of periostin mRNA (16). Periostin cDNA was introduced into T24 cells by retrovirus vector. As shown in Fig. $4 \mathrm{C}$ and D, introduction of periostin significantly suppressed cell invasiveness of T24 cells. The effect of siRNAs targeting human TAK1 and TAB1 on cell invasiveness was also examined in T24 cells expressing retroviral periostin. As shown in Fig. 4A and B, siRNAs targeting human TAK1 and TAB1 had a marked knockdown effect on endogenous TAK1 and TAB1, respectively, in $\mathrm{T} 24$ cells expressing retroviral periostin. As shown in Fig. 4C-E, siRNA targeting TAK1 or TAB1 markedly inhibited the suppression of cell invasiveness of T24 cells expressing retroviral periostin. These results indicate that TAK1 and TAB1 are also required for the suppression of cell invasiveness by periostin in human bladder cancer cells.

\section{Discussion}

In the present study, we identified TAB1, an activator of TAK1, as a periostin binding protein. Co-transfection experiments indicated that periostin, TAB1 and TAK1 are able to form complex. Periostin enhanced phosphorylation of TAK1 in co-transfected 293T cells. Knockdown of endogenous TAB1 suppressed TAK1 activation by periostin. The C-terminal region of periostin was necessary and sufficient for both the binding to TAB1 and activation of TAK1. Knockdown experiments by siRNA for TAB1 and TAK1 demonstrated that TAB1 and TAK1 are required for the suppression of cell invasiveness by periostin in both 293T and T24 bladder cancer cells. From these findings, we concluded that periostin is involved in the regulation of cell invasiveness via TAB1/ TAK1.

Periostin has been considered to be a secreted protein that acts as an adhesion molecule to associate with $\alpha_{v} \beta_{3}$ and $\alpha_{v} \beta_{5}$ integrins $(11,19)$. This was based on indirect experiments using a blocking assay by anti-integrin antibodies. However, we have previously proposed that periostin has a dual function, 
one as a secreted cell adhesion molecule and another as a cytoplasmic regulator of anchorage-independent growth and cell invasiveness of cancer cells $(16,18,20)$. In our immunoprecipitation assay in $293 \mathrm{~T}$ cells, TAB1 was identified as a cytosolic protein that associates with periostin, suggesting that this protein is involved in the regulation of cell invasiveness by periostin in the cytoplasm. In fact, the C-term mutant of periostin, which lacks the signal peptide region in the $\mathrm{N}$ terminus and the four RDs conserved in the fasciclin I family of adhesion molecules, could associate with TAB1 and activate TAK 1 in $293 \mathrm{~T}$ cells, suggesting that TAB1/TAK1 is involved in the suppression of cell invasiveness by periostin as cytosolic regulators. Knockdown experiments by siRNA for TAB1 and TAK1 supported this idea. In experiments with deletion mutants of periostin, the $\Delta \mathrm{N}$ mutant could bind to TAB 1 as well as WT periostin, but activation of TAK1 by the $\Delta \mathrm{N}$ mutant was moderate compared with that by WT or $\mathrm{C}$-term periostin. The $\mathrm{N}$-terminal region containing the signal peptide may also partially contribute to activation of TAK1 although it is not necessary for the binding to TAB1.

TAK1 is known to mediate various intracellular signaling pathways, such as p38 MAPK, c-jun NH2-terminal kinase (JNK), and IKK $(26,27)$. TAK1 has been also shown to be involved in the regulation of cell invasion and metastasis. In breast cancer, TAK1 promoted the in vitro cell invasiveness via the IKK signaling pathway $(28,29)$. In human breast cancer, periostin expression has also been shown to be upregulated although it was not detected in the normal tissues (11). Although these results may appear opposite to our findings in human bladder cancer, activation of TAK 1 is considered to be correlated with the expression of periostin in both cancer types. Just as the role of periostin in carcinogenesis may be different depending on the specific tissues and cell types (18), TAK1 activation by periostin may also occur differently in different types of cancers.

We have shown that periostin is able to associate with TAB1/TAK1 and that TAB1 and TAK1 are necessary for suppression of cell invasiveness by periostin. However, the mechanism of TAK1 activation by periostin via TAB1 still remains to be elucidated. There are two possible explanations. The first is that periostin might inhibit phosphorylation of TAB 1 to activate TAK1, because TAB1, which associates with p38 $\alpha$, negatively regulates TAK1 kinase activity by phosphorylating TAB1 at Ser 423, Thr 431, and Ser 438 (30). We have tested this possibility by examining the effect of periostin expression on the phosphorylation of TAB1 (Ser423) in $293 \mathrm{~T}$ cells. However, we could not detect any alteration of TAB 1 phosphorylation by periostin in our experimental conditions (data not shown). The second possibility is that periostin contributes to the stability of the TAB1/TAK1 complex to activate TAK1. Results of co-transfection experiments (Figs. 1B and 2A) indicated that the expression of TAB 1 enhances the expression level of TAK1 protein. Knockdown experiment by TAB1 siRNA also showed that $\mathrm{TAB} 1$ is required to maintain the expression level of TAK1 protein. Fig. 2A also indicated that the expression level of TAB 1 is also enhanced by TAK1. These findings suggest that the complex formation of TAB 1 and TAK1 is required to maintain and/or enhance the stability of both proteins. The triple complex formed by the binding of periostin to the
TAB1/TAK1 complex may also increase the stability of TAB1/TAK1 and enhance the phosphorylation of TAK1 to activate it. Further experiments will be necessary to clarify the molecular mechanism by which periostin activates TAK1 via TAB1 and suppresses cell invasiveness of bladder cancer cells. In the present study, we found that periostin is able to associate with TAB 1 to activate TAK 1 in the regulation of the cell invasiveness of bladder cancer cells. Investigations of TAB1/TAK 1 activation by periostin will also contribute to the elucidation of the role of periostin in bone and tooth formation, heart regulation, and other physiological functions.

\section{Acknowledgements}

We would like to thank Hiroko Kita (Division of Microbiology and Infectious Diseases, Shiga University of Medical Science) and Noboru Urushiyama (Central Research Laboratory, Shiga University of Medical Science) for their technical assistance. This project was supported by a Grant-in-Aid for Scientific Research (C) (Grants nos. 19591843, 17591675 and 17590341, to C.J. Kim, T. Isono and H. Inoue, respectively) from the Ministry of Education, Science, Sports, and Culture of Japan.

\section{References}

1. Horiuchi K, Amizuka N, Takeshita S, et al: Identification and characterization of a novel protein, periostin, with restricted expression to periostium and periodontal ligament and increased expression by transforming growth factor B. J Bone Miner Res 14: 1239-1249, 1999.

2. Takeshita S, Kikuno R, Tezuka K and Amann E: Osteoblastspecific factor 2: cloning of a putative bone adhesion protein with homology with the insect protein fasciclin I. Biochem J 294: 271-278, 1993.

3. Katsuragi N, Morishita R, Nakamura N, et al: Periostin as a novel factor responsible for ventricular dilation. Circulation 110: 1806-1813, 2004.

4. Wang D, Oparil S, Feng JA, et al: Effects of pressure overload on extracellular matrix expression in the heart of the atrial natriuretic peptide-null mouse. Hypertension 42: 88-95, 2003.

5. Kuhn B, del Monte F, Hajjar R, et al: Periostin induces proliferation of differentiated cardiomyocytes and prootes cardiac repair. Nat Med 13: 962-969, 2007.

6. Oka T, Xu J, Kaiser RA, et al: Genetic manipulation of periostin expression reveals a role in cardiac hypertrophy and ventricular remodeling. Circ Res 101: 313-321, 2007.

7. Wilde J, Yokozeki M, Terai K, Kudo A and Moriyama K: The divergent expression of periostin mRNA in the periodontal ligament during experimental tooth movement. Cell Tissue Res 312: 345-351, 2003.

8. Rios H, Koushik SV, Wang H, et al: Periostin null mice exhibit dwarfism, incisor enamel defects, and an early-onset periodontal disease-like phenotype. Mol Cell Biol 25: 11131-11144, 2005.

9. Kudo Y, Siriwardena BS, Hatano H, Ogawa I and Takata T: Periostin: novel diagnostic and therapeutic target for cancer. Histol Histopathol 22: 1167-1174, 2007.

10. Bao S, Ouyang G, Bai X, et al: Periostin potently promotes metastatic growth of colon cancer by augmenting cell survival via the Akt/PKB pathway. Cancer Cell 5: 329-339, 2004.

11. Shao R, Bao S, Bai X, et al: Acquired expression of periostin by human breast cancers promotes tumor angiogenesis through upregulation of vascular endothelial growth factor 2 expression. Mol Cell Biol 24: 3992-4003, 2004.

12. Sasaki H, Lo KM, Chen LB, et al: Expression of periostin, homologous with an insect cell adhesion molecule, as a prognostic marker in non-small cell lung cancers. Jpn J Cancer Res 92: 869-873, 2001.

13. Kudo Y, Ogawa I, Kitajima S, et al: Periostin promotes invasion and anchorage-independent growth in the metastatic process of head and neck cancer. Cancer Res 66: 6928-6935, 2006. 
14. Siriwardena BS, Kudo Y, Ogawa I, et al: Periostin is frequently overexpressed and enhances invasion and angiogenesis in oral cancer. Br J Cancer 95: 1396-1403, 2006.

15. Baril P, Gangeswaran R, Mahon PC, et al: Periostin promotes invasiveness and resistance of pancreatic cancer cells to hypoxiainduced cell death: role of the 34 integrin and the PI3k pathway. Oncogene 26: 2082-2094, 2007.

16. Kim CJ, Yoshioka N, Tambe Y, et al: Periostin is downregulated in high grade bladder cancer and suppresses in vitro cell invasiveness and in vivo metastasis of cancer cells. Int J Cancer 117: 51-58, 2005.

17. Shimagaki $\mathrm{M}$ and Kudo A: Impaired capsule formation of tumors in periostin-null mice. Biochem Biophys Res Commun 367: 736-742. 2008

18. Kim CJ, Isono T, Tambe Y, et al: Role of alternative splicing of periostin in human bladder carcinogenesis. Int J Oncol 32: 161-169, 2008.

19. Gillan L, Matei D, Fishman DA, et al: Periostin secreted by epithelial ovarian carcinoma is a ligand for alpha(V)beta(3) and alpha(V)beta(5) integrins and promotes cell motility. Cancer Res 62: 5358-5364, 2002.

20. Yoshioka N, Fuji S, Shimakage M, et al: Suppression of anchorage-independent growth of human cancer cell lines by the TRIF52/Periostin/OSF-2 gene. Exp Cell Res 279: 91-99, 2002.

21. Sakurai H, Miyoshi H, Toriumi W and Sugita T: Functional interactions of transforming growth factor beta-activated kinase 1 with IkappaB kinase to stimulate NF-kappaB activation. J Biol Chem 274: 10641-10648, 1999.

22. Naviaux RK, Constanzi E, Haas M and Verma IM: The pCL vector system: rapid production of helper-free, high-titer, recombinant retroviruses. J Virol 70: 5701-5705, 1996.
23. Singhirunnusorn P, Suzuki S, Kawasaki N, Saiki I and Sakurai H: Critical roles of threonine 187 phosphorylation in culture stressinduced rapid and transforming growth factor-beta-activated kinase 1 (TAK1) in a signaling complex containing TAK1binding protein TAB1 and TAB2. J Biol Chem 280: 7359-7368, 2004.

24. Isono T, Tanaka T, Kageyama S and Yoshiki T: Structural diversity of cancer-related and non-cancer-related prostatespecific antigen. Clin Chem 48: 2187-2194, 2002.

25. Shibuya H, Yamaguchi K, Shirakabe K, et al: TAB 1: an activator of the TAK1 MAPKKK in TGF-beta signal transduction. Science 272: 1179-1182, 1996.

26. Yamaguchi K, Shirakabe K, Shibuya H, et al: Identification of a member of the MAPKKK family as a potential mediator of TGF-beta signal transduction. Science 270: 2008-2011, 1995.

27. Sakurai H, Shigemori N, Hasegawa K and Sugita T: TGFbeta-activated kinase 1 stimulates NF-kappa B activation by an NF-kappa B-inducing kinase-independent mechanism. Biochem Biophys Res Commun 243: 545-549, 1998

28. Neil JR and Schiemann WP: Altered TAB1:I kappaB kinase interaction promotes transforming growth factor beta-mediated nuclear factor-kappaB activation during breast cancer progression. Cancer Res 68: 1462-1470, 2008.

29. Wada T and Penninger JM: Mitogen-activated protein kinase in apoptosis regulation. Oncogene 23: 2838-2849, 2004.

30. Cheng PC, Cambell DG, Nabreda AR and Cohen P: Feedback control of the protein kinase TAK 1 by SAPK2a/p38alpha. EMBO J 22: 5793-5805, 2003. 